\title{
Freehand diffuse optical spectroscopy imaging for intraoperative identification of major venous and arterial vessels underlying peritoneal fat: an in vivo demonstration in a pig model
}

Daqing Piao

Mohammad Ramadan

Aaron Park

Kenneth E. Bartels

Sanjay G. Patel 


\section{Freehand diffuse optical spectroscopy imaging for intraoperative identifica- tion of major venous and arterial vessels underlying peritoneal fat: an in vivo demonstration in a pig model}

\author{
Daqing Piao, a,b,* Mohammad Ramadan, ${ }^{\text {,dd }}$ Aaron Park, \\ Kenneth E. Bartels, ${ }^{b}$ and Sanjay G. Patel ${ }^{c}$ \\ aOklahoma State University, School of Electrical and Computer \\ Engineering, Stillwater, Oklahoma, United States \\ ${ }^{\mathrm{b}}$ Oklahoma State University, Department of Veterinary Clinical \\ Sciences, Center for Veterinary Health Sciences, Stillwater, \\ Oklahoma, United States \\ 'University of Oklahoma Health Sciences Center, Department of \\ Urology, Oklahoma City, Oklahoma, United States \\ dOklahoma City Veterans Affairs Health Care System, Department of \\ Urology, Oklahoma City, Oklahoma, United States \\ eOklahoma School of Science and Mathematics, Oklahoma City, \\ Oklahoma, United States
}

Abstract. Inadvertent injury to important anatomic structures is a significant risk in minimally invasive surgery (MIS) that potentially requires conversion to an open procedure, which results in increased morbidity and mortality. Surgeons operating minimal-invasively currently do not have an easy-to-use, real-time device to aid in intraoperative identification of important anatomic structures that underlie tissue planes. We demonstrate freehand diffuse optical spectroscopy (DOS) imaging for intraoperatively identifying major underlying veins and arteries. An applicator probe that can be affixed to and detached from an 8-mm laparoscopic instrument has been developed. The 10-mm DOS source-detector separation renders sampling of tissue heterogeneities a few millimeters deep. DOS spectra acquired consecutively during freehand movement of the applicator probe on the tissue surface are displayed as a temporal and spectral image to assist in spatially resolved identification of the underlying structures. Open surgery identifications of the vena cava and aorta underlying peritoneal fat of $\sim 4 \mathrm{~mm}$ in thickness using the applicator probe under room light were demonstrated repeatedly in multiple pigs in vivo. () The Authors. Published by SPIE under a Creative Commons Attribution 3.0 Unported License. Distribution or reproduction of this work in whole or in part requires full attribution of the original publication, including its DOI. [DOI: 10.1117/1.JBO.22.10.100503]

Keywords: minimally invasive surgery; diffuse optical spectroscopy; endoscopy; functional near-infrared spectroscopy.

Paper 170487LRR received Jul. 27, 2017; accepted for publication Oct. 4, 2017; published online Oct. 30, 2017.

*Address all correspondence to: Daqing Piao, E-mail: daqing.piao @ okstate.edu
Inadvertent injury to key anatomic structures, including major vasculature, in minimally invasive surgery (MIS) is a significant risk that potentially requires conversion to open surgery ${ }^{1}$ and results in increased morbidity ${ }^{2}$ and mortality. ${ }^{3}$ The concern of unintended injury to important underlying anatomic structures also potentially increases operative time in MIS, which in turn lengthens a patient's exposure to anesthesia, and increases overall cost. The concern of iatrogenic injury in MIS becomes even greater for obese patients because the large layer of peritoneal fat further challenges intraoperative definition of the internal anatomy that is already inherently difficult for MIS. ${ }^{4}$ Currently, surgeons operating minimal-invasively do not have an easy-touse, real-time device to aid in intraoperative identification of important anatomic structures that underlie native tissue planes ${ }^{5}$ or secondary scar tissues ${ }^{6}$ that must be carefully dissected in a stepwise layer-by-layer fashion to avoid injury to these structures. Techniques with a goal of detecting arterial vessels using standalone laparoscopic probes have been a topic of investigation for many years. ${ }^{7-10}$ All of these techniques (sensing blood pulsation mechanically, electrically, or optically) may provide information similar to the finger-based palpation used in open surgery that could become sensitive for identifying arterial vessels; however, sensing the pulsation alone is inherently ineffective for identifying low- or nonpulsatile vessels such as veins, and for other key structures, including ureters, bile ducts, and intestines. Any configuration of the probing technology that requires a standalone probe to be operated by the surgeon by first releasing control of the MIS tissue-manipulating instruments in the operative field to detect arterial vessels will also impede adaptation of the technique for a clinical procedure.

In this letter, we demonstrate a simple method of freehand diffuse optical spectroscopy imaging (freeDOSi) for intraoperatively identifying significant anatomic structures, including major veins and arteries underlying the plane of dissection for targeted applications in MIS. Diffuse optical spectroscopy (DOS) sampling of tissue heterogeneities underlying a tissue surface of probe contact using a pair of distantly placed source and detector channels on the probe is well known ${ }^{11}$ and has been used experimentally for identifying blood vessels in a laboratory frequency-domain open space configuration. ${ }^{12}$ However, what makes the present demonstration uniquely pertinent is the development of an applicator probe that can be affixed, detached, resterilized, and reapplied for additional use with a standard $(8 \mathrm{~mm})$ laparoscopic instrument for intraoperative DOS sampling of underlying major venous and arterial vessel structures. Further, given that the dynamic responses of DOS to underlying tissue heterogeneities at sufficient levels of signal-to-noise ratios are the foundations of many approaches, such as near-infrared spectroscopy monitoring of cerebral hemodynamics, ${ }^{13}$ what makes the present approach innovative and robust is the conversion of consecutive dynamic DOS profiles acquired during freehand movement of the probe over the dissection surface to a twodimensional temporal and spectral image that intuitively and spatially correlates with the anatomic course of underlying tissue structures presenting the detected spectral heterogeneity.

Figure 1 shows the laparoscopically adaptable freeDOSi applicator probe. The freeDOSi applicator probe has an 8-mm-inner lumen allowing insertion of an 8-mm da Vinci Endowrist ${ }^{\circledR}$ instrument and setting of the applicator probe at any position along the uniform shaft of the 8-mm Endowrist ${ }^{\circledR}$ instrument. The freeDOSi applicator probe has an outer diameter of $12 \mathrm{~mm}$ 


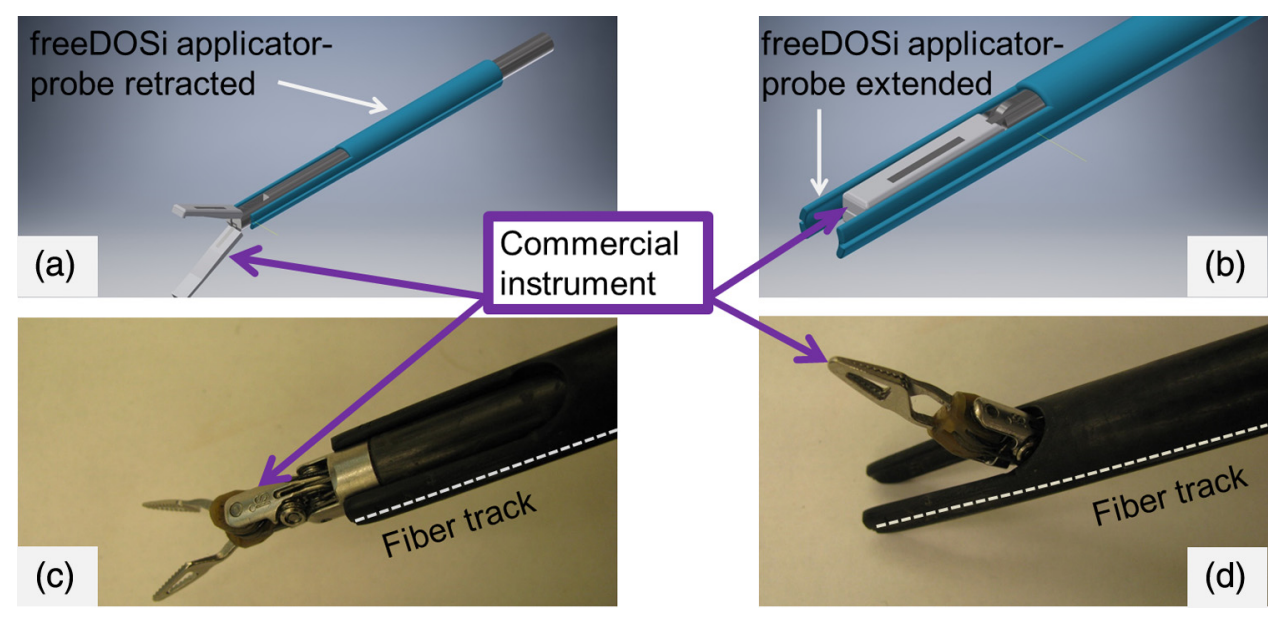

Fig. 1 The laparoscopically adaptable applicator probe for freeDOSi operation. (a) Three-dimensional (3-D) rendering of the applicator probe when retracted along the shaft of a tissue-manipulating instrument for insertion and extraction prior to and after tissue dissection. (b) 3-D rendering of the applicator probe when extended along the shaft of a tissue-manipulating instrument for tissue contact. (c) The actual applicator probe retracted along an 8-mm laparoscopic instrument. (d) The actual applicator probe extended for tissue contact along an 8-mm laparoscopic instrument.

for passing through a 12-mm trocar port. The freeDOSi applicator probe has two grooves in the rounded shell structure placed 180 deg opposite to each other, extending to the end of the probe designed for tissue contact. In each of the two grooves on the applicator probe, a $400-\mu \mathrm{m} 0.37 \mathrm{NA}$ fiber of $3 \mathrm{~m}$ in length is optically epoxied, ensuring a $10-\mathrm{mm}$ separation between the two fiber tips that are shielded from each other by shell tips located medial to the fibers. The two fibers of $3 \mathrm{~m}$ in length are coupled at the instrument ends to either a light source or a compact spectrometer through SMA-905 termination. The freeDOSi system using the laparoscopically adaptable applicator probe was integrated on a heavy duty cart (Anthro Convoi) for operating room readiness. An ultrabright broadband laserdriven light source (LDLS) (EQ-99XFC, Energetiq Inc., Woburn, Massachusetts) was used for tissue illumination by coupling the $400-\mu \mathrm{m}$ fiber source channel of the applicator probe through a $230-\mu \mathrm{m} 0.22$ NA fiber. The $400-\mu \mathrm{m}$ fiber detector channel of the applicator probe was connected to a compact 16-bit spectrometer (USB4000-VIS-NIR, Ocean Optics, Largo, Florida) with a customized slit and sensor configuration. The effective spectral response of the system was 530 to $1000 \mathrm{~nm}$. The data acquisition from the spectrometer was controlled by a customuser interface developed in LabVIEW (National Instruments, Austin, Texas).

A reference signal from a calibrated phantom was acquired by placing the applicator probe in a 2-in. diameter assembly that held and aligned the probe to allow the source-detector of the probe to be in direct contact with an industrial tissue-mimicking phantom (Biomimic Optical Phantom, PB0335, INO, Québec, Canada). Limited testing of identifying underlying tubular heterogeneities embedded in a diffuse medium of controlled properties was performed by laterally translating a tubular inclusion embedded in a $1 \%$ bulk intralipid solution, as shown in the photograph of Fig. 2(a). A plastic tubing with a diameter of $4 \mathrm{~mm}$ and a wall thickness of $0.5 \mathrm{~mm}$ (approximate dimensions of medium size artery or vein) was embedded at an edge depth of $2 \mathrm{~mm}$ corresponding to a tubing-center depth of $\sim 4.5 \mathrm{~mm}$. The tubing was filled by red, blue, or green food coloring dye (Assorted Food Color \& Egg Dye, Wal-Mart Stores Inc, Bentonville, Arkansas) at a concentration of $33.3 \%$, respectively, or filled with water or air only. The lateral position of the tubing with respect to the applicator probe was adjusted at a step of $0.5 \mathrm{~mm}$ by a translation stage. The tubing was placed at a total of 41 lateral positions covering a total of $20 \mathrm{~mm}$, with the center position corresponding to the tubing placed exactly below the applicator probe. The DOS profiles were acquired under room light at an acquisition time of $100 \mathrm{~ms}$. The noise profiles (obtained by placing the applicator probe onto a black cloth free of ambient lighting) were removed from the DOS profiles obtained from any test medium prior to normalization with respect to a reference profile obtained from the aforementioned calibration phantom. The freeDOSi images formed by the consecutive normalized DOS profiles acquired at the 20-mm lateral range over the 41 positions are presented in Fig. 2(b). Each freeDOSi image represents a temporal and spectral map of DOS profiles. The ordinate of the 2-D image corresponds to a relative position between the underlying tubing and the probe, and the abscissa of the 2-D image reveals a spectral discrepancy between the targeted tissue structure and the calibration reference phantom. A concentrated strip of spectral contrast, thereby, visualizes the crossover of the sensitive volume of the probe sampling geometry with the anatomic course of an underlying tissue structure presenting the spectral discrepancy with respect to the phantom of spectral reference. Any underlying tissue heterogeneity in the tubing that is less absorbing than the reference phantom, such as air or water, consequently presents positive spectral contrast over the entire spectral range, whereas any tissue heterogeneity that has characteristically strong absorbance, such as dye, presents a significant negative contrast in their respective characteristic spectral bands. The time versus spectral intensity distribution of Fig. 2(b) isolated at a 575$\mathrm{nm}$ wavelength is plotted in one-dimension in Fig. 2(c) to clarify the bipolar spectral contrasting associated with freeDOSi for the less absorbing air or water and the more absorbing dye contents of the same underlying tubular heterogeneity.

FreeDOSi was tested in vivo in a porcine model in the University of Oklahoma Health Sciences Center with the approval of the Institutional Animal Care and Use Committee (\#10148916-017-NS). Although the applicator probe for freeDOSi could be affixed to an 8-mm Endowrist ${ }^{\circledR}$ tissue-manipulating instrument 


\section{JBO Letters}
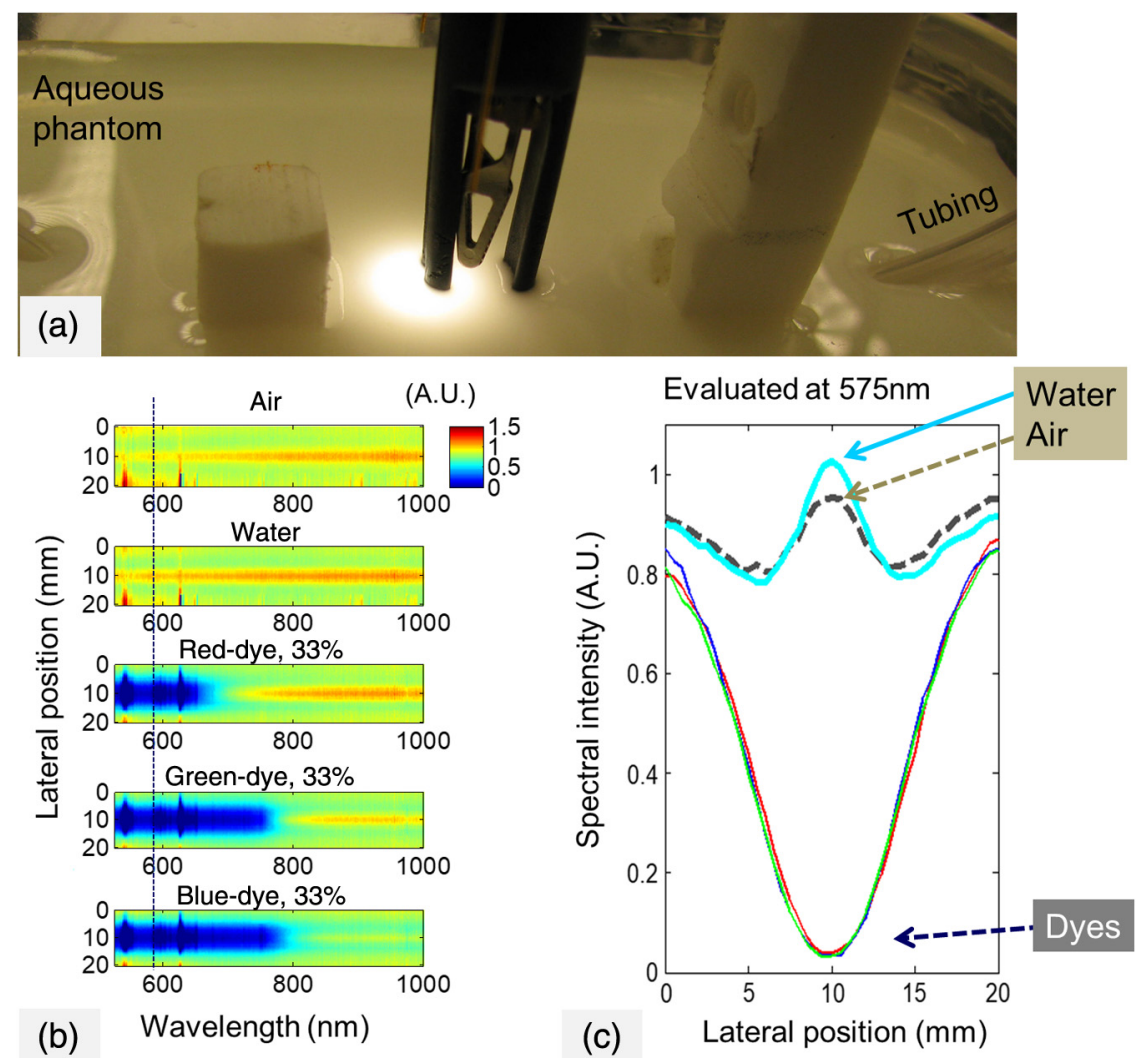

Fig. 2 (a) Laboratory configuration for testing freeDOSi performance in identifying underlying tubular heterogeneities embedded in a diffuse medium of controlled properties. A plastic tubing with a diameter of $4 \mathrm{~mm}$ and a wall thickness of $0.5 \mathrm{~mm}$ was embedded in the aqueous tissue phantom using a structure affixed to a translation stage for adjusting the lateral position of the tubing with respect to the applicator probe. (b) The freeDOSi images corresponding to 41 consecutive DOS profiles acquired as the applicator probe were scanned laterally at a step of $0.5 \mathrm{~mm}$ with respect to the 4-mm tubing immerged at a 2-mm edge depth from the surface of the $1 \%$ intralipid medium. The top image was for the tubular content of air. The second image from the top was for the tubing filled with water. The three images at the bottom half were for the tubing filled with $33 \%$ red dye, 33\% green dye, and 33\% blue dye, respectively. (c) The 1-D profile across the freeDOSi images of (b) at $575 \mathrm{~nm}$.

for robotically assisted control, the in vivo testing using the pig models was performed in an open surgery fashion to minimize ambiguities and eliminate technical difficulties in demonstrating the principle of freeDOSi. Female adult cross breed Yorkshire pigs were used in this study. After anesthesia was induced, the animals were secured supine on the operating table. A 15- to 20$\mathrm{cm}$ midline incision was used to expose the abdominal cavity. After the vena cava and aorta were located and exposed, a layer of adipose tissue up to $4 \mathrm{~mm}$ in thickness was dissected from the peritoneum with the blood supply maintained, sutured to the connective tissue in the periphery of the vena cava and aorta complex such that the caudal half section of the exposed vena cava and aorta complex was covered as shown in Fig. 3(a). The cranial half section of the vena cava and aorta complex was exposed as shown in Fig. 3(b) for visual identification of the vena cava and aorta. The freeDOSi applicator probe was placed in contact with the tissue covering the vena cava and aorta complex, with the source-detector oriented along the cranial to caudal direction. Using a flexible plastic ruler positioned perpendicular to the anatomic course of the vena cava and aorta complex but parallel to the movement of the applicator probe, the freeDOSi applicator probe was manually scanned laterally over the adipose tissue area covering the vena cava and aorta complex as shown in Fig. 3(c). The DOS spectrum was recorded every $5 \mathrm{~mm}$ at a total of 11 positions covering a length of $50 \mathrm{~mm}$. The DOS profiles from the pigs were acquired with a spectrometer exposure time of $300 \mathrm{~ms}$. The overhead operative surgery light was directed away from the surgical field. The procedure was repeated by one of two surgeons for multiple measurements taken from each of four pigs. The duplicative procedures resulted in freeDOSi images that were all similar to the one presented in Fig. 3(d). The image contains a linear band of hyperabsorption over the entire spectral band of interest. The lateral center of the hyperabsorptive band corresponds to the geometric axis of vena cava, and the lateral expansion of the hyperabsorptive band is greater than the size of vena cava. The image also contains a band of hypoabsorption over the partial spectral band of interest (600 to $900 \mathrm{~nm}$ ). The lateral peak of the hypoabsorptive band roughly corresponds to the geometric axis of aorta whereas the lateral expansion of the hypoabsorptive band is greater than the size of aorta. The one-dimensional (1-D) profile in Fig. 3(e) corresponds to the DOSi measurements over the $40-\mathrm{mm}$ length but is isolated at $700 \mathrm{~nm}$. The hyperabsorptive and hypoabsorptive features of the DOS profiles acquired respectively at the positions corresponding to the vena cava and aorta are clearly revealed in Fig. 3(e).

In conclusion, we have demonstrated a simple method of freeDOSi for intraoperatively identifying significant underlying 
(a)

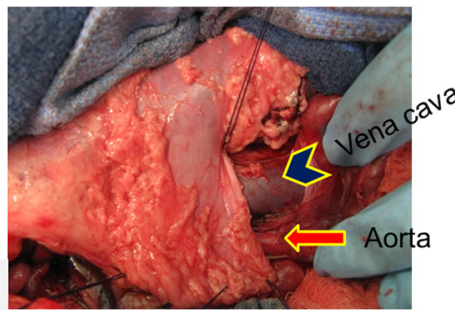

(b)

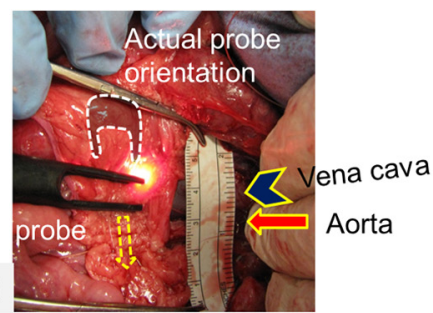

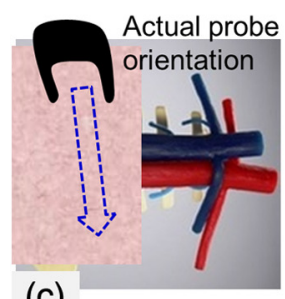

(c)
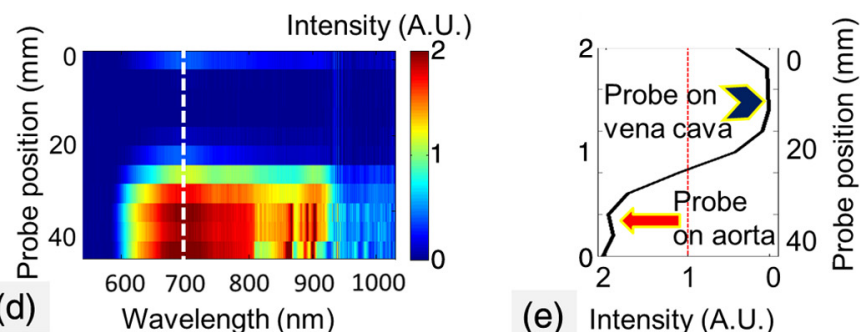

(e) Intensity (A.U.)

Fig. 3 (a) The caudal half section of the vena cava and aorta complex was covered by a layer of adipose tissue. The wire for suturing is seen above the vena cava. (b) A flexible ruler was placed over the exposed cranial half section of the vena cava and aorta complex. The applicator probe with the two tips aligned medial to lateral was placed by the ruler for appreciation of the relative scales. (c) The applicator probe was placed with the two tips aligned cranial to caudal when moved on the adipose tissue covering the vena cava and aorta complex for freeDOSi operation. (d) One example of the freeDOSi image. (e) A 1-D profile of the freeDOSi image of (d) at $700 \mathrm{~nm}$.

anatomic structures, including major veins and arteries. An applicator probe that can be affixed to and detached from an 8-mm laparoscopic instrument for operative procedures using existing tissue-manipulating instruments has been developed. The 10-mm source-detector separation renders diffuse sampling of tissue heterogeneities a few millimeters deep. Consecutively acquired DOS spectra during freehand movement of the applicator probe on tissue surface have been displayed to form a temporal and spectral image to assist in spatially resolved identification of underlying structures presenting DOS heterogeneity. Open surgery identifications of the vena cava and aorta underlying peritoneal fat of $\sim 4 \mathrm{~mm}$ in thickness under room light have been demonstrated repeatedly in multiple pigs in vivo.

\section{Disclosures}

D. Piao and K. E. Bartels have ownership interests in Surgiotonics LLC, which did not sponsor this work. Other authors declare no conflicts of interest.

\section{Acknowledgments}

This work was supported in part by a grant (\#P2016-0048) from Oklahoma State University Technology Development Center.

\section{References}

1. V. Sharma and J. J. Meeks, "Open conversion during minimally invasive radical prostatectomy: impact on perioperative complications and predictors from national data," J. Urol. 192, 1657-1662 (2014).

2. W. T. Lowrance et al., "Costs of medical care after open or minimally invasive prostate cancer surgery: a population-based analysis," Cancer 118, 3079-3086 (2012).
3. S. F. Montes et al., "Intraoperative laparoscopic complications for urological cancer procedures," World J. Clin. Cases 3, 450-456 (2015).

4. K. Yuge et al., "How does visceral obesity affect surgical performance in laparoscopic radical nephrectomy?" Jpn. J. Clin. Oncol. 45, 373-377 (2015).

5. R. F. Perini et al., "Isolated right segmental hepatic duct injury following laparoscopic cholecystectomy," Cardiovasc. Interv. Radiol. 28, 185195 (2005).

6. M. T. Siedhoff et al., "Robotic-assisted laparoscopic removal of cesarean scar ectopic and hysterotomy revision," Am. J. Obstet. Gynecol. 212, 681.e1-681.e4 (2015).

7. Z. Glavic, L. Begic, and R. Rozman, "A new device for the detection and recognition of blood vessels in laparoscopic surgery," Surg. Endosc. 16, 1197-1200 (2002)

8. S. McKinley et al., "A single-use haptic palpation probe for locating subcutaneous blood vessels in robot-assisted minimally invasive surgery," in IEEE Int. Conf. on Automation Science and Engineering, pp. 1151-1158 (2015).

9. J. Kim, "Non-invasively detecting blood vessels during laparoscopic/ robotic procedure," J. Biosens. Bioelectron. 7, 214 (2016).

10. A. Chaturvedi et al., "Optics based signal processing methods for intraoperative blood vessel detection and quantification in real time," Proc. SPIE 9707, 97070Q (2016).

11. D. A. Boas et al., "Detection and characterization of optical inhomogeneities with diffuse photon density waves: a signal-to-noise analysis," Appl. Opt. 36, 75-92 (1997).

12. D. Y. Paithankar, K. T. Schomacker, and N. S. Nishioka, "Frequencydomain optical detection of subsurface blood vessels: experimental and computational studies using a scattering phantom," IEEE J. Sel. Top. Quantum Electron. 5(4), 1032-1039 (1999).

13. H. Fahnenstich, "Near-infrared spectroscopy-a new method for noninvasive monitoring of cerebral hemodynamics," Klin Padiatr. 205, 398-403 (1993). 